\title{
A Chinese Patient with Pusher Syndrome and Unilateral Spatial Neglect Syndrome
}

\author{
Xiao-Wei Chen, Cheng-He Lin, Hua Zheng, Zhen-Lan Li
}

\begin{abstract}
Objective: To observe clinical manifestations, behavioral characteristics, and effects of rehabilitation on a patient with pusher syndrome and unilateral spatial neglect caused by right thalamic hemorrhage. Methods: Assessment of pusher syndrome was made by the Scale for Contraversive Pushing (SCP), and unilateral spatial neglect syndrome was diagnosed using line cancellation, letter and star cancellation, line bisection tests and copy and continuation of graphic sequence test. Behavioral therapy, occupational therapy, reading training and traditional Chinese medicine methods were adopted for treatment of pusher syndrome and unilateral spatial neglect. Results: The patient showed typical pusher syndrome and unilateral spatial neglect symptoms. The pusher syndrome and unilateral spatial neglect symptoms were significantly improved following rehabilitation treatments. Conclusions: Pusher syndrome and unilateral spatial neglect syndrome occurred simultaneously after right thalamic hemorrhage. Early rehabilitation therapy can reduce the symptoms of pusher syndrome and unilateral spatial neglect syndrome and improve motor function.
\end{abstract}

\begin{abstract}
RÉSUMÉ: Un patient chinois atteint du syndrome du Pusher et du syndrome de négligence spatiale unilatérale. Objectif : Le but de l'étude était d'observer les manifestations cliniques, les caractéristiques de comportement et les résultats de la réadaptation chez un patient atteint du syndrome de pusher et du syndrome de négligence spatiale unilatérale suite à une hémorragie thalamique droite. Méthode : L'évaluation du syndrome de pusher a été effectuée au moyen du Scale for Contraversive Pushing (SCP) et le syndrome de négligence spatiale unilatérale a été diagnostiqué au moyen des tests de bisection de ligne, de barrage de lettres et d'étoiles et de dysgraphie spatiale. Le patient a été traité par thérapie comportementale, ergothérapie, entrainement à la lecture et par des méthodes de médecine chinoise traditionnelle pour son syndrome de pusher et son syndrome de négligence spatiale unilatérale. Résultats : Le patient présentait un syndrome de pusher et des symptômes de négligence spatiale unilatérale typiques. Le syndrome de pusher et les symptômes de négligence spatiale unilatérale se sont améliorés significativement suite à la réadaptation. Conclusions : Le syndrome de pusher et le syndrome de négligence spatiale unilatérale sont survenus simultanément après une hémorragie thalamique droite. La réadaptation précoce peut diminuer les symptômes du syndrome de pusher et du syndrome de négligence spatiale unilatérale et améliorer la motricité.
\end{abstract}

Can J Neurol Sci. 2014; 41: 493-497

Pusher syndrome is a clinical disorder characterized by a particular behavioral pattern in stroke patients with hemiplegia. Generally, hemiplegia patients incline their body to the healthy side in order to keep balance, while patients with the pusher syndrome always actively push away from the non-hemiparetic side and resist passive correction to the midline of the body. ${ }^{1}$ There are also patients who tilt their body backwards and resist passive correction forwards, ${ }^{2}$ leading to impaired balance that can affect the ability to walk. Unilateral spatial neglect refers to patients with brain injury who are unable to realize things in contralateral space, which significantly affects the recovery of motor function and quality of life. This condition also impairs processing of visual cues, the patient disregarding the side of space contralateral space to the lesion. However, it is unclear whether the mechanism of this disability is primarily due to sensory, motor or emotional impairment. ${ }^{3}$ Pusher syndrome or neglect has been reported widely. However, cases with patients presenting with both pusher syndrome or neglect syndrome were seldom reported. In this case report, we observed a patient with both pusher syndrome and unilateral spatial neglect caused by right thalamic hemorrhage, which provides a clinical basis for the pathogenesis of simultaneous concurrence of pusher syndrome and unilateral spatial neglect.

\footnotetext{
From the Rehabilitation Medical Center, the First Hospital of Jilin University, Changchun, P. R. China.

Received December 5, 2013. Final Revisions Submitted February 25, 2014. Correspondence to: Zhen-Lan Li, Rehabilitation Medical Center, the First Hospital of Jilin University, Changchun, 130021, P.R. China. Email: lizhenlan1306040@163.com, zhenlanli66@163.com.
} 


\section{Methods}

\section{Patient}

Li, a 55-year old, right-handed illiterate female was admitted to the Department of Rehabilitation on February 20, 2012 due to disability of the left limb. Physical examination showed the following: blood pressure was $140 / 90 \mathrm{mmHg}$, normal cognition, normal speech without facial apraxia and limb apraxia, but with shallow left nasolabial, lolling middle, upward mobility of the left eye. Normal vision and hearing were assessed by coarse measurement, without hemianopia. Left hemiplegia was observed. The left reflex-quads were normal, the left ankle jerk was increased, and ankle clonus was classified as level 1 (3 second clonus). There was little spasticity in the lower limb. The sensory modalities of proprioception, vibration, and light touch were normal. A decreased response to pain stimulation was detected. Mini mental status examination (MMSE) score was 22 points. According to the Brunnstrom classification, ${ }^{4}$ the left upper limb-hand-lower limb was III-IV-III degree. Fugl-Meyer scores $^{5}$ were 170 points. The total movement score in this patient was 64 points (the upper limb was 38 points, the lower limb was 26 points). The total balance score was 5 points, the total sensory score was 15 points, the total range of motion score was 43 points, and the total pain score was 43 points (Full Fugl-Meyer scores: 100 points for movement, 66 points for the upper limb, 34 points for the lower limb; 14 points for balance; 24 points for sensory intact; 44 points for range of motion; 44 points for pain).

The Barthel index for measurement of performance in activities of daily living was 40 points (full score is 100 points). A computer tomography (CT) scan revealed high density flakes in the right side of the thalamus, at a maximum level of $3.2 \times 2.4$ $\mathrm{cm}$. Bilateral ventricular enlargement was observed. Enlargement of the left ventricle was more evident than that of the right ventricle. Similar high density was observed in the third and fourth ventricule (Figure 1). No obvious shift was seen in midline structures. Clinical diagnosis of the right side of the thalamus hemorrhage suggested an intra-ventricular hemorrhage.

One month after the initial examination, when the patient came to the Department for Rehabilitation Therapy, she tilted to the affected side whenever in sitting or standing position, and only ate food on the right of a plate when dining. We evaluated her based on assessment standards of pusher syndrome and unilateral spatial neglect, and diagnosis of the pusher syndrome and unilateral spatial neglect were made after the assessment. A one-month rehabilitation treatment was initiated, and we found that motor function in this patient was markedly improved. The patient was able to walk independently under supervision. During the patient's return visit on May 25, 2012, although she could walk independently, fine motor function of her left hand was comprised such that she could not button her own shirt. We concluded that pusher syndrome had completely disappeared, and that unilateral spatial neglect syndrome was significantly improved. According to the Brunnstrom classification, the left upper limb-hand-lower limb was V-V-VI grade. Fugl-Meyer scores were 215 points: the total movement score was 94 points (the score in the upper limb was 62 points, the score in the lower limb was 32 points), the total balance score was 11 points, the total sensory score was 24 points, the total range of motion score was 43 points, the total pain score was 43 points. The Barthel index was 100 points. Ethic approval of this study was provided by the Clinical Research Ethics Committee in our hospital.

\section{Evaluation Methods}

\section{Evaluations of Pusher syndrome ${ }^{6,7}$}

Scale for Contraversive Pushing (SCP) was applied for evaluation of the Pusher syndrome. The scale contains three parts: (A): degree of symmetry of posture: 1 , severe to suffering from side to side tilt and fall; 0.75 , severe to suffering from side tilt, no fall; 0.25 , mild to suffering from side tilt, no fall; 0 , the body is erect, without inclination; (B): the presence of abduction or extension of the uninvolved extremities to increase the area of contact with the supporting surface: 1 , when the rest is; 0.5 , only the change in posture; 0 points, without stretching; $(\mathrm{C})$ : the degree of resistance to passive postural correction: 1, a resistance; 0 , without resistance. Each part is classified 0-1 point,

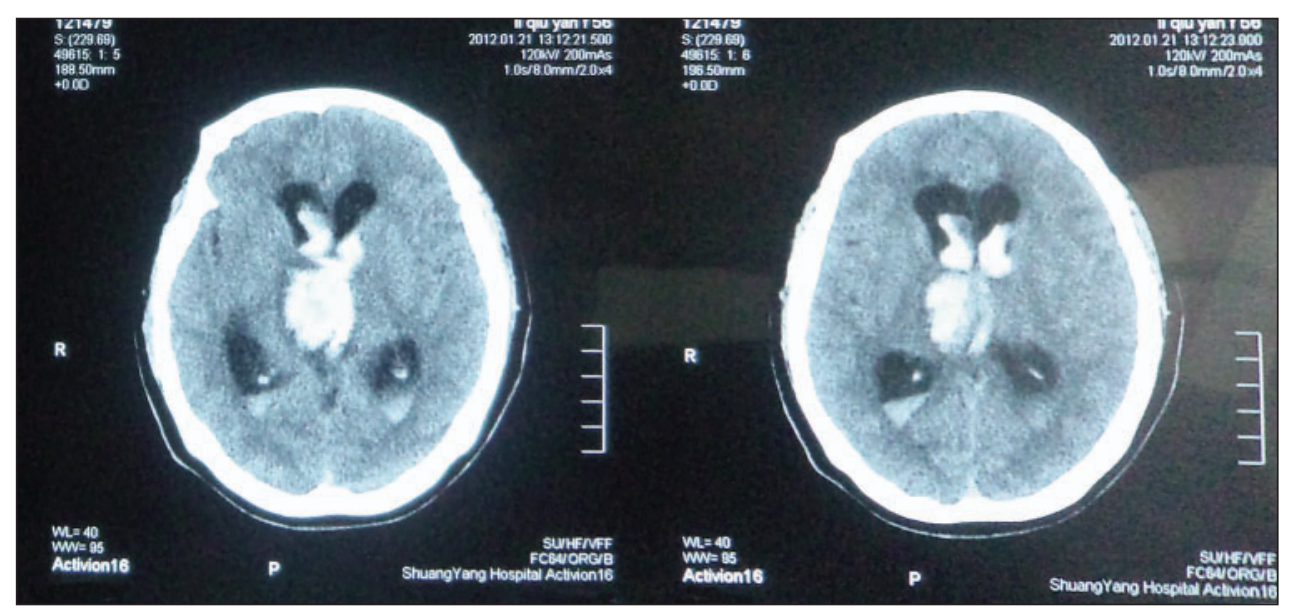

Figure 1: A respresentative CT scan image: A high density area was seen on the right side of the thalamus, at the maximum level of $3.2 \times 2.4 \mathrm{~cm}, C T$ value $67 \mathrm{HU}$. Bilateral ventricular enlargement was visible, more evident at the left side. Similar high density was observed in the fourth and third ventricles. 
tested in both sitting and standing positions, the highest score is 6 points. If each result was $\geq 1$ point (total scores $\geq 3$ points), pusher syndrome was diagnosed.

\section{Unilateral spatial neglect assessments}

Several tests were selected from a series of behavioral neglect tests (Behavioral Inattention Test, BIT) ${ }^{8}$ :

(A) Line cancellation test: the patient was given a piece of paper with 40 lines, each line was $25 \mathrm{~mm}$ long (18 in the left space, 18 in the right space, 4 in the middle). The paper was placed just in front of the patient. The patient was asked to cross all lines on the paper using a pen. Numbers that were crossed in each space were recorded.

(B) Letter cancellation test: the patient was given a piece of paper with letters which were arranged in five rows, 34 letters in each row, the letters $E$ and $R$ were placed across the whole page (20 in the left space: 10 letters for $E$ and 10 letters for $\mathrm{R}$ in the left space; 20 in the right space: 10 letters for $\mathrm{E}$ and 10 letters for $\mathrm{R}$ in the right space), the patient was asked to find all $\mathrm{E}$ and $\mathrm{R}$ on the paper, and the number of $\mathrm{E}$ or $\mathrm{R}$ that were found in each space were recorded.

(C) Star cancellation test: the patient was given a piece of paper with big stars, letters and words which were arranged randomly, along with 56 small stars diffused on the paper (27 in the left space, 27 in the right space, 2 in the middle). The patient was asked to cross all the small stars on the paper, and the number of $\mathrm{E}$ or $\mathrm{R}$ that were crossed in each space were recorded.

(D) Line bisection test: the patient was given a piece of paper with three parallel, horizontal lines, each line of which was 2.04 $\mathrm{cm}$ long, the patient was asked to mark the midpoint of each line. The length was measured from a point marked to the actual midpoint.

(E) Copy and continuation of graphic sequence test: the patient was asked to copy two symmetric graphics, the shape of the copied graphics was observed.

\section{Treatments}

This patient underwent one course of rehabilitation treatment which lasted for 30 days.

\section{Treatments for Pusher syndrome}

Correction of the patient's abnormal posture was made by visual feedback technology, including restoration of head movement, maintaining full range of motion of the neck, guiding active movement of the head and keeping the head in the normal position. Training of shifting center of gravity was performed, including transferring weight to the non-paralyzed side in sitting and standing positions, transitting to maintain the center of gravity in the neutral position gradually, recovery of midline standing, improving the muscle strength of the paralyzed lower limb, practicing correct standing posture, and walking was performed under supervision. A mirror was used during training to remind the patient to correct their posture. Training was performed 40 minutes per day, five days a week, three weeks in total. Walk training was performed by the lower limb robot for 20 minutes daily.

\section{Treatment for unilateral spatial neglect}

\section{Nursing}

Stimulation of neglected side was performed by allowing the patient to lie in right lateral supine position. Family members or medical staff stood on the neglected side of the patient when they communicated with the patient. The patient was encouraged to complete activities of daily living using the affected side as much as possible.

\section{Occupational therapy}

Attention to the neglected side was accomplished by marking the edge of the neglected side with a bright color when completing visual scanning training to increase sensory stimulation of the neglected side. The patient received training such as verbal, visual, tapping therapy, and electric stimulation of sensory input. This task training focused on crossing the midline from the healthy side to the ignored side for 40 minutes daily.

\section{Reading training}

Anchoring technology ${ }^{9}$ provides a visual cue to the neglected side, which helped the patient find a starting point of reading. However, as the patient improved, the visual cue was reduced. Training lasted for 20 minutes daily.

\section{Acupuncture therapy}

Fu et $a l^{10}$ have shown that scalp acupuncture has a curative effect for some cerebral diseases. The effects were especially noticable in improving the motor function and mobility of patients with unilateral spatial neglect by promoting cerebral collateral circulation, and improving neural function defects induced by cerebral ischemia and hypoxia . In this case, several acupuncture points were chosen, including Baihui, Yintang, Fengchi, Jianyu, Quchi, Waiguan, Hegu, Yanglingquan, Taichong, Zulinqi. Acupuncture was performed for 15 minutes daily by an Acupuncturist at the Department of Rehabilitation, the First Hospital of Jilin University.

\section{RESUlTS}

\section{Evaluation results of Pusher syndrome prior to treatments}

Prior to treatment, we observed that the head of the patient shifted to the right side when sitting, and the trunk leaned to the left. She returned to her original (incorrect) position after she was corrected to the neutral position.

Spatial neglect was observed on the left side, as exemplified by the finding that when family members or a therapist talked on her left side, she had no response.

When transferred from bed to chair, the patient could not stretch her right leg, which was often accompanied by tilting of her body backwards. The patient also could not stand upright because of obvious leaning towards the left. The total SCP was 5 points (A, 1 point; B, 2 points; C, 2 points).

\section{Evaluation results of unilateral spatial neglect prior to treatment}

The results of line cancellation test showed that 10 out of 18 lines were crossed in the left space (10/18), 4 out of 4 lines in the 
middle were crossed (4/4), 18 out of 18 lines in the right space were crossed (18/18), these results indicated that spatial neglect occurred on the left side.

The results of the letter cancellation test revealed that the number of letter $E$ in the left space was 8/10 (10 means 10 letters for $\mathrm{E}$ on the left space, and 8 means 8 of the 10 letters were deleted), and the number of letter $\mathrm{E}$ in the right space was $9 / 10$. The number of letter $\mathrm{R}$ in the left space was $6 / 10$, and the number of letter $\mathrm{R}$ in the right space was $9 / 10$.

When the patient performed the star cancellation test, the number of stars she crossed in the left space was 19 out of 27 , the crossed number in the middle was 2 out of 2 , and the crossed number in the right space was 26 out of 27.

After the patient marked the midpoint of three lines, the results of the line bisection test revealed that $\mathrm{R}$ ( $\mathrm{R}$ means the distance between the midpoint which the patient marked and the real midpoint) of three lines were $-0.2 \mathrm{~cm}, 0.1 \mathrm{~cm}$, and $3 \mathrm{~cm}$. As shown in Figure 2, the patient had difficulty in copying standard graphics provided.

\section{Evaluation results of Pusher syndrome after treatments}

After treatment, we found that the patient had no obvious shift in supine position, sitting and standing. The visual feedback in the left space improved, and she had normal response when family members or therapists talked to her on the left side. Moreover, she had no difficultly in transferring from sitting to standing, and she could steadily stand upright. Her SCP after treatment was 1 point (A, 0 point; $B, 0$ point; $C, 1$ point: had little resistance to passive postural correction in standing position).

\section{Curative effect of rehabilitation therapy after treatment}

After one month of rehabilitation, patient mobility markedly increased without a noticable incline in any positions. Upon discharge, she could walk independently under supervision. Three months later, fine motor function of her left hand improved, Pusher syndrome had been completely corrected, and unilateral spatial neglect significantly improved.

\section{Discussion}

Pusher syndrome is a disorder which manifests as severe postural control impairment after stroke. The incidence of pusher syndrome is $23 \%$ in Chinese patients with cerebrovascular disease, ${ }^{11}$ other statistical data is $5 \%-63 \%$ in patients from other countries. ${ }^{6}$ The difference in statistical data may be associated with different diagnostic criteria and selected populations. ${ }^{12}$

Although many pusher syndrome studies have been published in recent years, morbidity in hospitalized patient and prognosis of comprehensive rehabilitation treatment is seldom studied. Pusher syndrome presents after stroke and, in many cases, can be corrected within six months; however, the phenotype is occasionally persistent. ${ }^{13}$ It is thought that the prevalence of pusher syndrome in right cerebral hemisphere-damaged patients is higher than in left cerebral hemisphere-damaged patients. ${ }^{14}$ Another study showed that pusher syndrome is related to impairment of sensory pathways results from damage of the posterior limb of the internal capsule; however, this cause has not yet been confirmed. ${ }^{6}$ Santos-Pontelli et al ${ }^{15}$ used neuroimaging scans to locate lesions in the anterior and posterior crus

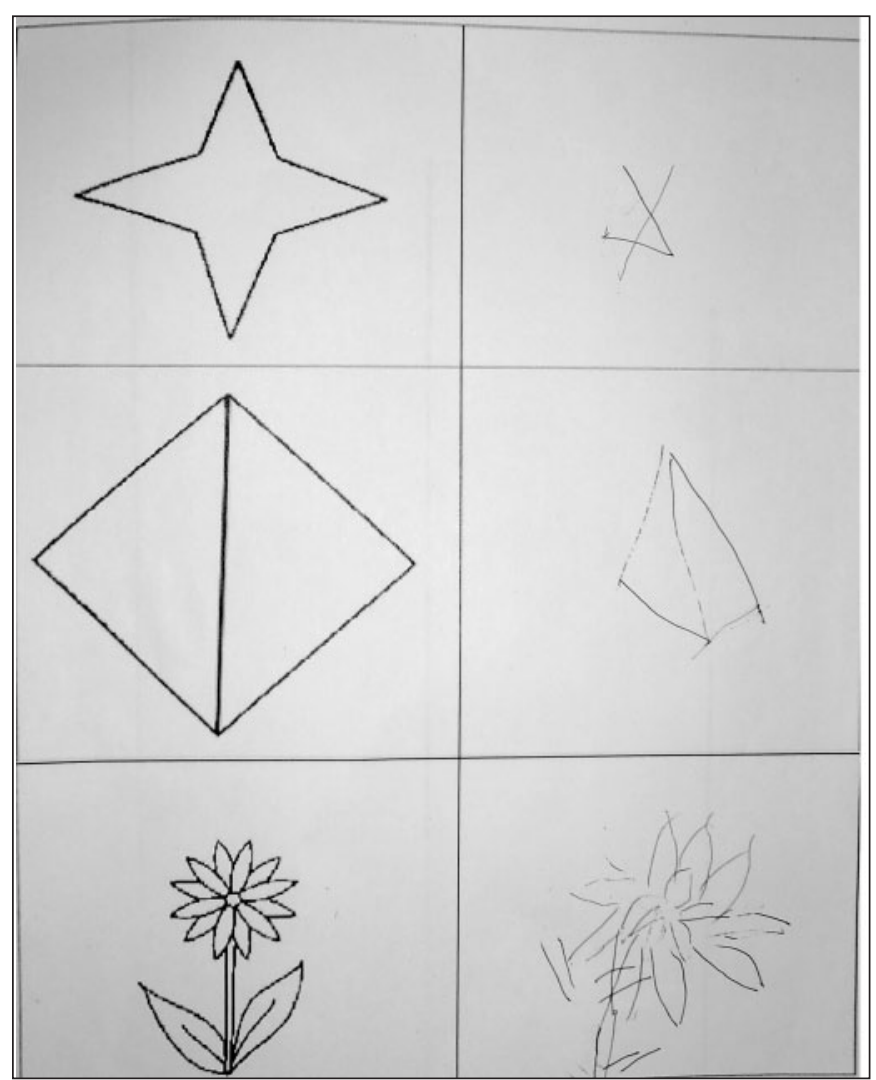

Figure 2: First row: A star cancellation test; Second row: A line bisection test; Third row: A graphics copy test. Left column: standard test; Right column: the patient cannot appropriately copy the images on the left or with structure errors.

of the internal capsule, thalamus, frontal, temporal and parietal lobes, insula and hemispheric lesions in patients with pusher syndrome. In addition, damage in cortical and subcortical regions, such as the insular cortex and posterior central gyrus, have also been suggested to cause pusher syndrome. ${ }^{16}$ Using functional MRI, Ticini et $a l^{13}$ confirmed that hypoperfusion of the inferior frontal gyrus, middle temporal gyrus and inferior parietal lobe affected postural control. These researchers suggest that hypoperfusion may be causal in pusher syndrome. ${ }^{13}$ Moreover, Baier et al found when the right hemisphere was impaired, the insula, superior temporal gyrus and posterior tectal zones had been identified to be associated with pusher syndrome. When the left hemisphere is injured, the insular front, top cover area and projection fibers to the lateral thalamus inner portion of the sac were believed to be associated with pusher syndrome. These brain areas are associated with postural control, or related to processing of vestibular information. ${ }^{17}$

Currently, the pathogenesis of pusher syndrome is still unclear. Patients with pusher syndrome need much longer time to recover motor function, compared to patients without pusher syndrome. ${ }^{18}$ Therefore, earlier diagnosis and treatment of pusher syndrome can effectively facilitate the recovery of mobility and shorten hospitalization time. ${ }^{19}$ Studies have reported that visual feedback training, which corrects subjective vertical sense 
through visual input, is beneficial to control symptoms of pusher syndrome..$^{20,21}$

It has been shown that gait training assisted by lower limb robotics increases proprioceptive input of body upright position, and improves pusher symptoms through the improvement of postural control. ${ }^{22}$ Stimulation of the vestibular sensory input channel by direct electrical stimulation of the vestibular system improves subjective vertical sense, thereby it controls the patient's body inclining it to the normal position. ${ }^{23}$ Krewer et al found that effect of lower limb robotic assisted ambulation training is more remarkable than visual feedback training in randomized double-blind trials, and that direct electrical stimulation of the vestibular system has no significant effect. ${ }^{12}$ The current research uses visual feedback training and gait training to correct pusher symptoms at an early phase of pusher syndrome. This approach has been demonstrated to promote the patient's independent walking ability and shorten the time of hospitalization.

Patients $(56.3 \%)$ with pusher syndrome have unilateral spatial neglect, while the remaining patients without pusher syndrome were presented with agnosia, apraxia and other neurocognitive dysfunctions, suggesting that pusher syndrome and unilateral spatial neglect may be two separate syndromes, and only appear to have casual correlation with each other. ${ }^{10}$ Davies et al has reported that pusher syndrome caused by left hemisphere damage had no association with unilateral spatial neglect. ${ }^{1}$ In contrast, Karnath asserts that patients with pusher syndrome are often associated with unilateral spatial neglect when there are lesions in the right thalamus, pusher syndrome is accompanied with aphasia if lesions are on the left side. ${ }^{24}$ In the patients with right hemisphere injury, unilateral spatial neglect and pusher syndrome are highly correlated. However, there is no evidence to support the hypothesis that pusher syndrome is caused by unilateral spatial neglect, because there are still $20 \%$ of patients with pusher syndrome who have no unilateral spatial neglect when the right hemisphere is damaged.

We present the first report of a patient with right thalamic hemorrhage presenting with both pusher syndrome and unilateral spatial neglect. We think that early rehabilitation treatment can have a significant beneficial effect on disease progression that shortens the time of hospitalization. This case provides clinical evidence for further study into the pathogenesis and therapy of pusher syndrome with unilateral spatial neglect.

A limitation of this study is the inadequate number of patients, which limits the ability of this study to explain the correlations between the lesion site and pusher syndrome with unilateral spatial neglect. Further collection of additional cases and appropriate study design are necessary to explore the pathogenesis.

\section{ACKNOWLEDGEMENT}

The authors thank Medjaden Bioscience Limited for assisting in the preparation of this manuscript.

\section{REFERENCES}

1. Davies PM. Steps to follow. The comprehensive treatment of patients with hemiplegia. 2nd ed. Berlin: Springer; 2000. p. $402-8$
2. Cardoen S, Santens P. Posterior pusher syndrome: a report of two cases. Clin Neurol Neurosurg. 2010;112(4):347-9.

3. Chen XW, Shan CL, Li ZL. Performance of different sensory modalities and spatial frames of reference and the effect of rehabilitation in a patient with unilateral spatial neglect symptom. Chin Cerebrovasc Dis. 2012;9(6):317-20.

4. Brunnstrom S. Movement therapy in hemiplegia: a neurophysiological approach. New York: Harper \& Row; 1970. p. 34-55.

5. Fugl-Meyer AR, Jääskö L, Leyman I, Olsson S, Steglind S. The post-stroke hemiplegic patient. 1. A method for evaluation of physical performance. Scand J Rehabil Med. 1975;7(1):13-31.

6. Karnath HO, Ferber S, Dichgans J. The origin of contraversive pushing: evidence for a second graviceptive system in humans. Neurology. 2000;55(9):1298-304.

7. Baccini M, Paci M, Nannetti L, Biricolti C, Rinaldi LA. Scale for contraversive pushing: cutoff scores for diagnosing "pushing behavior" and construct validity. Phys Ther. 2008;88(8):947-55.

8. Wilson BA, Cockburn J, Halligan PW. Behavioural inattention test. England: Thames Valley Test Company; 1987.

9. Jacques L, Peter H, Gilles R. Visuo-spatial neglect: a systematic review of current interventions and their effectiveness. Neurosci Biobehav Rev. 2006;30(7):961-82.

10. Fu JM, Gu DX, Yao YH, Shi MF, Zhu MH. Clinical observation on curative effect of unilateral spatial neglect caused by stroke treated with scalp acupuncture for long- time retenting needle and rehabititation. Chin J Trad Med Sci Technol. 2011;18(1): $53-4$.

11. Liu SW, An XF, Song HC. A study on symptom of Pusher phenomenon. Chin J Rehabil Med. 1998;13(2):62-5.

12. Krewer C, Rieß K, Bergmann J, Müller F, Jahn K, Koenig E. Immediate effectiveness of single-session therapeutic interventions in pusher behavior. Gait Posture. 2013;37(2): 246-50.

13. Ticini LF, Klose U, Nägele T, Karnath HO. Perfusion imaging in Pusher syndrome to investigate the neural substates involved in controlling upright body position. PloS One. 2009;4(5):e5737.

14. Abe H, Kondo T, Oouchida Y, Suzukamo Y, Fujiwara S, Izumi S. Prevalence and length of recovery of Pusher syndrome based on cerebral hemispheric lesion side in patients with acute stroke. Stroke. 2012;43(6):1654-6.

15. Santos-Pontelli TE, Pontes-Neto OM, Araujo DB, Santos AC, Leite JP. Neuroimaging in stroke and non-stroke pusher patients. Arq Neuropsiquiatr. 2011;69(6):914-19.

16. Karnath HO, Suchan J, Johannsen L. Pusher syndrome after ACA territory infarction. Eur J Neurol. 2008;15(8):e84-5.

17. Baier B, Janzen J, Müller-Forell W, Fechir M, Müller N, Dieterich M. Pusher syndrome: its cortical correlate. J Neurol. 2012;259 (2):277-83

18. Krewer C, Luther M, Müller F, Koenig E. Time course and influence of pusher behavior on outcome in a rehabilitation setting: a prospective cohort study. Top Stroke Rehabil. 2013;20 (4):331-9.

19. Paci M, Baccini M, Rinaldi LA. Pusher behaviour: a critical review of controversial issues. Disabil Rehabil. 2009;31(4):249-58.

20. Broetz D, Johannsen L, Karnath HO. Time course of "pusher syndrome" undervisual feedback treatment. Physiother Res Int. 2004;9(3):138-43.

21. Paci M, Nannetti L. Physiotherapy for pusher behaviour in a patient with post-stroke hemiplegia. J Rehabil Med. 2004;36(4):183-5.

22. Luther M, Krewer C, Müller F, Koenig E. Wirkung der LokomatTherapie auf die Pusher-Symptomatik. (in German) Aktuelle Urol. 2007;34(10):S147.

23. Saj A, Honore J, Rousseaux M. Perception of the vertical in patients with right hemispheric lesion: effect of galvanic vestibular stimulation. Neuropsychologia. 2006;44(8):1509-12.

24. Karnath HO, Johannsen L, Broetz D, Küker W. Posterior thalamic hemorrhage induces "pusher syndrome". Neurology. 2005;64 (6):1014-19. 